2019 4th International Conference on Information Technology, Information Systems and Electrical Engineering (ICITISEE)

EICITISEE

took place 20-21 November 2019 in Yogyakarta, Indonesia.

$\begin{array}{ll}\text { IEEE catalog number: } & \text { CFP19G48-ART } \\ \text { ISBN: } & 978-1-7281-5118-2\end{array}$

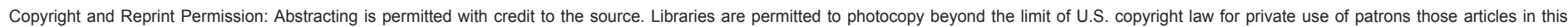

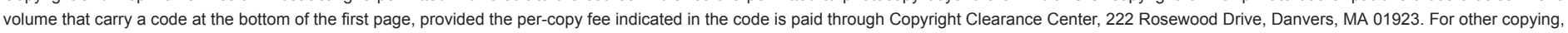
reprint or republication permission, write to IEEE Copyrights Manager, IEEE Operations Center, 445 Hoes Lane, Piscataway, NJ 08854 . All rights reserved. Copyright $\odot 2019$ by IEEE. 


\section{Technical Program Committee}

\begin{tabular}{|c|c|c|}
\hline Intan Ermahani A. Jalil & Universiti Teknikal Malaysia Melaka & Malaysia \\
\hline Mohd Helmy Abd Wahab & Universiti Tun Hussein Onn Malaysia & Malaysia \\
\hline MS. Hendriyawan Achmad & Yogyakarta University of Technology & Indonesia \\
\hline Sumarni Adi & Universitas AMIKOM Yogyakarta & Indonesia \\
\hline I Adiyasa & Gadjah Mada University & Indonesia \\
\hline I Made Agastya & Universitas Amikom Yogyakarta & Indonesia \\
\hline Mohd Khairul Ikhwan Ahmad & Universiti Tun Hussein Onn Malaysia & Malaysia \\
\hline Ali Qusay Al-Faris & Assumption College & USA \\
\hline Shajith Ali & SSN College of Engineering, Chennai & India \\
\hline Gede Angga Pradiptha & Institut Teknologi dan Bisnis STIKOM Bali & Indonesia \\
\hline Igi Ardiyanto & Universitas Gadjah Mada & Indonesia \\
\hline Dhani Ariatmanto & Universitas Amikom Yogyakarta & Indonesia \\
\hline Agus Aribowo & Universitas Pembangunan Nasional Veteran Yogyakarta & Indonesia \\
\hline Andria Arisal & Indonesian Institute of Sciences & Indonesia \\
\hline Arti Arya & PESIT-Bangalore South Campus & India \\
\hline Ika Astuti & Universitas Amikom Yogyakarta & Indonesia \\
\hline Hasyim Asyari & Universitas Muhammadiyah Surakarta & Indonesia \\
\hline Azizul Azizan & Universiti Teknologi Malaysia (UTM) & Malaysia \\
\hline Aslina Baharum & Universiti Malaysia Sabah & Malaysia \\
\hline Aashish Bardekar & Sipna College of Engineering and Technology, Amravati & India \\
\hline Agus Bejo & Universitas Gadjah Mada & Indonesia \\
\hline Adha Imam Cahyadi & Universitas Gadjah Mada & Indonesia \\
\hline Alessandro Carrega & CNIT & Italy \\
\hline Maria Chiara Caschera & CNR & Italy \\
\hline Selem Charfi & HD Technology & France \\
\hline Wichian Chutimaskul & King Mongkut's University of Technology Thonburi & Thailand \\
\hline Domenico Ciuonzo & University of Naples Federico II, IT & Italy \\
\hline Akhmad Dahlan & Universitas Amikom Yogyakarta & Indonesia \\
\hline
\end{tabular}




\begin{tabular}{|c|c|c|}
\hline Ni Ketut Dewi Ari Jayanti & Institute of Technology and Business STIKOM Bali & Indonesia \\
\hline Andi Wahju Rahardjo Emanuel & Universitas Atma Jaya Yogyakarta & Indonesia \\
\hline Ahmad Fajar & Bina Nusantara University & Indonesia \\
\hline Muhammad Faris & Universitas Gadjah Mada & Indonesia \\
\hline Hanif Fatta & Universitas AMIKOM Yogyakarta & Indonesia \\
\hline Anggit Ferdita Nugraha & Universitas AMIKOM Yogyakarta & Indonesia \\
\hline Alireza Ghasempour & ICT Faculty & USA \\
\hline Arifiyanto Hadinegoro & Amikom Universty Yogyakarta & Indonesia \\
\hline Byeong-jun Han & Soongsil University & Korea (South) \\
\hline Muh Hanafi & Universitas Amikom Yogyakarta & Indonesia \\
\hline Seng Hansun & Universitas Multimedia Nusantara & Indonesia \\
\hline Hartatik Hartatik & STMIK AMIKOM Yogyakarta & Indonesia \\
\hline Mardhiya Hayaty & Universitas AMIKOM Yogyakarta & Indonesia \\
\hline Purwono Hendradi & Universitas Muhammadiyah Magelang & Indonesia \\
\hline Roberto Carlos Herrera Lara & National Polytechnic School & Ecuador \\
\hline Indriana Hidayah & Universitas Gadjah Mada & Indonesia \\
\hline Tonny Hidayat & Universitas AMIKOM Yogyakarta & Indonesia \\
\hline Nor Hikmah & Universitas AMIKOM & Indonesia \\
\hline Danial Hooshyar & Korea University & Korea (South) \\
\hline Hozairi Hozairi & Islamic University of Madura & Indonesia \\
\hline Imelda Imelda & Universitas Budi Luhur & Indonesia \\
\hline Rini Indrayani & Universitas Amikom Yogyakarta & Indonesia \\
\hline Nurulisma Ismail & Universiti Malaysia Perlis & Malaysia \\
\hline Iswandi Iswandi & Gadjah Mada University & Indonesia \\
\hline Ramkumar Jaganathan & VLB Janakiammal College of Arts and Science & India \\
\hline Arihant Jain & Jaipur Engineering College \& Research Centre & India \\
\hline Biao Jiang & The City University of New York & USA \\
\hline Dimitrios Kallergis & University of West Attica & Greece \\
\hline Sandy Kosasi & STMIK Pontianak & Indonesia \\
\hline
\end{tabular}




\begin{tabular}{|c|c|c|}
\hline Krisnawati Krisnawati & University of AMIKOM Yogyakarta & Indonesia \\
\hline Ryan Kristianto & Universitas Amikom Yogyakarta & Indonesia \\
\hline Sumit Kushwaha & KNIT Sultanpur & India \\
\hline Kusnawi Kusnawi & AMIKOM University & Indonesia \\
\hline Kusrini Kusrini & AMIKOM Yogyakarta University & Indonesia \\
\hline Pavel Loskot & Swansea University & United Kingdom (Great Britain) \\
\hline Emha Taufiq Luthfi & Universitas AMIKOM Yogyakarta & Indonesia \\
\hline Mahdin Mahboob & Stony Brook University & USA \\
\hline Robert Marco & STMIK Amikom Yogyakarta & Indonesia \\
\hline Prita Dewi Mariyam & Universitas Indonesia & Indonesia \\
\hline Ahlihi Masruro & Universitas AMIKOM Yogyakarta & Indonesia \\
\hline Ratheesh Kumar Meleppat & University of California Davis & USA \\
\hline Ahmed Mobashsher & The University of Queensland & Australia \\
\hline Seyed Sahand Mohammadi Ziabari & Vrije University of Amsterdam & The Netherlands \\
\hline Amrit Mukherjee & Jiangsu University & China \\
\hline I Wayan Mustika & Universitas Gadjah Mada & Indonesia \\
\hline Syibrah Naim & Universiti Sains Malaysia & Malaysia \\
\hline Warsun Najib & Universitas Gadjah Mada & Indonesia \\
\hline Asro Nasiri & University of Amikom Yogyakarta & Indonesia \\
\hline Shah Nazir & University of Peshawar & Pakistan \\
\hline Ruzelita Ngadiran & Universiti Malaysia Perlis & Malaysia \\
\hline Bhanu Nugraha & Universitas Amikom Yogyakarta & Indonesia \\
\hline Hanung Nugroho & Universitas Gadjah Mada & Indonesia \\
\hline Prapto Nugroho & Universitas Gadjah Mada & Indonesia \\
\hline Nitish Ojha & DIT University, Dehradun & India \\
\hline Ilker Ali Ozkan & Selcuk University & Turkey \\
\hline Oktavia Permata & Institut Teknologi Telkom Surabaya & Indonesia \\
\hline Anugerah Persada & Universitas Gadjah Mada & Indonesia \\
\hline Kiran Sree Pokkuluri & Shri Vishnu Engineering College for Women & India \\
\hline
\end{tabular}




\begin{tabular}{|c|c|c|}
\hline N. Prabaharan & SASTRA Deemed University & India \\
\hline Gede Pramudya Ananta & Universiti Teknikal Malaysia Melaka & Malaysia \\
\hline Andri Pranolo & Universitas Ahmad Dahlan & Indonesia \\
\hline Prihandoko Prihandoko & University of Gunadarma & Indonesia \\
\hline Yoga Pristyanto & Universitas Amikom Yogyakarta & Indonesia \\
\hline Reza Pulungan & Universitas Gadjah Mada & Indonesia \\
\hline Nila Puspitasari & Universitas AMIKOM Yogyakarta & Indonesia \\
\hline Guntur Putra & University of New South Wales & Australia \\
\hline Lesnanto Multa Putranto & UGM & Indonesia \\
\hline Ali Rafiei & University of Technology Sydney & Australia \\
\hline Suwanto Raharjo & Informatics of The Institut Sains \& Teknologi AKPRIND Yogyakarta & Indonesia \\
\hline Hemant Kumar Rath & Tata Consultancy Services & India \\
\hline Rizky Rizky & Universitas AMIKOM Yogyakarta & Indonesia \\
\hline Rumini Rumini & Universitas AMIKOM Yogyakarta & Indonesia \\
\hline G. p. Sajeev & Amrita Vishwa Vidyapeetham & India \\
\hline Andreas Sandiwan & Gadjah Mada University & Indonesia \\
\hline Banu Santoso & Universitas AMIKOM Yogyakarta & Indonesia \\
\hline Leo Santoso & Petra Christian University & Indonesia \\
\hline Sayantam Sarkar & Vijaya Vittala Institute of Technology & India \\
\hline Theopilus Bayu Sasongko & Universitas AMIKOM Yogyakarta & Indonesia \\
\hline Mithileysh Sathiyanarayanan & City, University of London & United Kingdom (Great Britain) \\
\hline Vaibhav Saundarmal & Marathwada Institute of Technology, Aurangabad & India \\
\hline Enny Sela & Universitas Teknologi Yogyakarta & Indonesia \\
\hline Erni Seniwati & Universitas AMIKOM Yogyakarta & Indonesia \\
\hline Anindita Septiarini & Univeristas Mulawarman & Indonesia \\
\hline Amel Serrat & USTO MB & Algeria \\
\hline Bayu Setiaji & Universitas AMIKOM Yogyakarta & Indonesia \\
\hline Noor Akhmad Setiawan & Universitas Gadjah Mada & Indonesia \\
\hline Emy Setyaningsih & Institute of Science \& Technology AKPRIND & Indonesia \\
\hline
\end{tabular}




\begin{tabular}{|c|c|c|}
\hline Arief Setyanto & Universitas AMIKOM Yogyakarta & Indonesia \\
\hline Iwan Setyawan & Satya Wacana Christian University & Indonesia \\
\hline Andik Setyono & Universitas Dian Nuswantoro & Indonesia \\
\hline Aditi Sharma & Quantum University, Roorkee, Uttarakhand & India \\
\hline Vesh Raj Sharma Banjade & Intel Corporation & USA \\
\hline Abdul Samad Shibghatullah & UCSI University & Malaysia \\
\hline Monika Sikri & Cisco Systems India Pvt Ltd. & India \\
\hline Amando Singun & Higher College of Technology, Muscat & Oman \\
\hline Achmad Solichin & Universitas Budi Luhur & Indonesia \\
\hline lickho Song & Korea Advanced Institute of Science and Technology & Korea (South) \\
\hline Yi-Jen Su & Shu-Te University & Taiwan \\
\hline Suhirman Suhirman & Universitas Teknologi Yogyakarta & Indonesia \\
\hline Andi Sunyoto & Universitas AMIKOM Yogyakarta & Indonesia \\
\hline Sutarman Sutarman & Faculty Of Information Technology and Business, University Technoloy of Yogyakarta & Indonesia \\
\hline Melwin Syafrizal & Universitas AMIKOM Yogyakarta & Indonesia \\
\hline Srinivasulu Tadisetty & Kakatiya University College of Engineering and Technology & India \\
\hline Chakib Taybi & Mohammed First University & Morocco \\
\hline Ivanna Timotius & Satya Wacana Christian University & Indonesia \\
\hline Evi Triandini & Institut Teknologi dan Bisnis STIKOM Bali & Indonesia \\
\hline Hastari Utama & Universitas Amikom Yogyakarta & Indonesia \\
\hline Ema Utami & Universitas Amikom Yogyakarta & Indonesia \\
\hline Dario Vieira & EFREI & France \\
\hline Anik Vitianingsih & Universitas Dr Soetomo & Indonesia \\
\hline Mochammad Wahyudi & Universitas Gadjah Mada & Indonesia \\
\hline Sri Ngudi Wahyuni & Universitas AMIKOM Yogyakarta & Indonesia \\
\hline Fikri Waskito & Universitas Gadjah Mada & Indonesia \\
\hline Leong Wen Chek & University of Malaya & Malaysia \\
\hline Sunu Wibirama & Universitas Gadjah Mada & Indonesia \\
\hline Adi Wibowo & Diponegoro University & Indonesia \\
\hline
\end{tabular}




\begin{tabular}{|c|c|c|}
\hline Ferry Wahyu Wibowo & Universitas Amikom Yogyakarta & Indonesia \\
\hline Sigit Wibowo & Gadjah Mada University & Indonesia \\
\hline Teguh Wibowo & Gadjah Mada University & Indonesia \\
\hline Ainul Yaqin & Universitas Amikom Yogyakarta & Indonesia \\
\hline Thaweesak Yingthawornsuk & King Mongkut's University of Technology Thonburi & Thailand \\
\hline Uky Yudatama & Universitas Indonesia & Indonesia \\
\hline Go Yun II & Heriot-Watt University Malaysia & Malaysia \\
\hline Muhammad Yusuf & University of Trunojoyo, Madura & Indonesia \\
\hline Sri Zuliana & UIN Sunan Kalijaga & Indonesia \\
\hline Nur Zareen Zulkarnain & Universiti Teknikal Malaysia Melaka & Malaysia \\
\hline
\end{tabular}




\section{Additional Reviewers}

\begin{tabular}{|c|c|c|}
\hline Intan Ermahani A. Jalil & Universiti Teknikal Malaysia Melaka & Malaysia \\
\hline Mohd Helmy Abd Wahab & Universiti Tun Hussein Onn Malaysia & Malaysia \\
\hline MS. Hendriyawan Achmad & Yogyakarta University of Technology & Indonesia \\
\hline I Wayan Adiyasa & Gadjah Mada University & Indonesia \\
\hline Ali Qusay Al-Faris & Assumption College & USA \\
\hline Shajith Ali & SSN College of Engineering, Chennai & India \\
\hline Gede Angga Pradiptha & Institut Teknologi dan Bisnis STIKOM Bali & Indonesia \\
\hline Dhani Ariatmanto & Universitas Amikom Yogyakarta & Indonesia \\
\hline Agus Sasmito Aribowo & Universitas Pembangunan Nasional Veteran Yogyakarta & Indonesia \\
\hline Andria Arisal & Indonesian Institute of Sciences & Indonesia \\
\hline Arti Arya & PESIT-Bangalore South Campus & India \\
\hline Aslina Baharum & Universiti Malaysia Sabah & Malaysia \\
\hline Maria Chiara Caschera & CNR & Italy \\
\hline Wichian Chutimaskul & King Mongkut's University of Technology Thonburi & Thailand \\
\hline Domenico Ciuonzo & University of Naples Federico II, IT & Italy \\
\hline Ni Ketut Dewi Ari Jayanti & Institute of Technology and Business STIKOM Bali & Indonesia \\
\hline Andi Wahju Rahardjo Emanuel & Universitas Atma Jaya Yogyakarta & Indonesia \\
\hline Ahmad Nurul Fajar & Bina Nusantara University & Indonesia \\
\hline Anggit Ferdita Nugraha & Universitas AMIKOM Yogyakarta & Indonesia \\
\hline Alireza Ghasempour & ICT Faculty & USA \\
\hline Seng Hansun & Universitas Multimedia Nusantara & Indonesia \\
\hline Hartatik Hartatik & STMIK AMIKOM Yogyakarta & Indonesia \\
\hline Mardhiya Hayaty & Universitas AMIKOM Yogyakarta & Indonesia \\
\hline Roberto Carlos Herrera Lara & National Polytechnic School & Ecuador \\
\hline Tonny Hidayat & Universitas AMIKOM Yogyakarta & Indonesia \\
\hline Danial Hooshyar & Korea University & Korea (South) \\
\hline Hozairi Hozairi & Islamic University of Madura & Indonesia \\
\hline Rini Indrayani & Universitas Amikom Yogyakarta & Indonesia \\
\hline Nurulisma Ismail & Universiti Malaysia Perlis & Malaysia \\
\hline Ramkumar Jaganathan & VLB Janakiammal College of Arts and Science & India \\
\hline Biao Jiang & The City University of New York & USA \\
\hline Sandy Kosasi & STMIK Pontianak & Indonesia \\
\hline Krisnawati Krisnawati & University of AMIKOM Yogyakarta & Indonesia \\
\hline Ryan Putranda Kristianto & Universitas Amikom Yogyakarta & Indonesia \\
\hline Sumit Kushwaha & KNIT Sultanpur & India \\
\hline Pavel Loskot & Swansea University & United Kingdom (Great Britain) \\
\hline Prita Dewi Mariyam & Universitas Indonesia & Indonesia \\
\hline Ratheesh Kumar Meleppat & University of California Davis & USA \\
\hline Amrit Mukherjee & Jiangsu University & China \\
\hline Syibrah Naim & Universiti Sains Malaysia & Malaysia \\
\hline Asro Nasiri & University of Amikom Yogyakarta & Indonesia \\
\hline Shah Nazir & University of Peshawar & Pakistan \\
\hline Ruzelita Ngadiran & Universiti Malaysia Perlis & Malaysia \\
\hline Hanung Adi Nugroho & Universitas Gadjah Mada & Indonesia \\
\hline Prapto Nugroho & Universitas Gadjah Mada & Indonesia \\
\hline Ilker Ali Ozkan & Selcuk University & Turkey \\
\hline Oktavia Ayu Permata & Institut Teknologi Telkom Surabaya & Indonesia \\
\hline Gede Pramudya Ananta & Universiti Teknikal Malaysia Melaka & Malaysia \\
\hline Prihandoko Prihandoko & University of Gunadarma & Indonesia \\
\hline Guntur Dharma Putra & University of New South Wales & Australia \\
\hline Lesnanto Multa Putranto & UGM & Indonesia \\
\hline Suwanto Raharjo & Informatics of The Institut Sains \& Teknologi AKPRIND Yogyakarta & Indonesia \\
\hline Bambang Riyono & Gadjah Mada University & Indonesia \\
\hline Leo Santoso & Petra Christian University & Indonesia \\
\hline Vaibhav Dudhaji Saundarmal & Marathwada Institute of Technology, Aurangabad & India \\
\hline Enny Sela & Universitas Teknologi Yogyakarta & Indonesia \\
\hline Ririn Septrisulviani & Universitas Gadjah Mada & Indonesia \\
\hline Amel Serrat & USTO MB & Algeria \\
\hline
\end{tabular}




\begin{tabular}{|l|l|l|}
\hline Lilis Susanti Setianingsih & Universitas Gadjah Mada & Indonesia \\
\hline Noor Akhmad Setiawan & Universitas Gadjah Mada & Indonesia \\
\hline Iwan Setyawan & Satya Wacana Christian University & Indonesia \\
\hline Abdul Samad Shibghatullah & UCSI University & Malaysia \\
\hline Achmad Solichin & Universitas Budi Luhur & Indonesia \\
\hline lickho Song & Korea Advanced Institute of Science and Technology & Korea (South) \\
\hline Andi Sunyoto & Universitas AMIKOM Yogyakarta & Indonesia \\
\hline Melwin Syafrizal & Universitas AMIKOM Yogyakarta & Indonesia \\
\hline Srinivasulu Tadisetty & Kakatiya University College of Engineering and Technology & India \\
\hline Chakib Taybi & Mohammed First University & Morocco \\
\hline Ivanna Timotius & Satya Wacana Christian University & Indonesia \\
\hline Evi Triandini & Institut Teknologi dan Bisnis STIKOM Bali & Indonesia \\
\hline Anik Vega Vitianingsih & Universitas Dr Soetomo & Indonesia \\
\hline Leong Wen Chek & University of Malaya & Malaysia \\
\hline Adi Wibowo & Diponegoro University & Indonesia \\
\hline Ainul Yaqin & Universitas Amikom Yogyakarta & Indonesia \\
\hline Thaweesak Yingthawornsuk & King Mongkut's University of Technology Thonburi & Thailand \\
\hline Uky Yudatama & Universitas Indonesia & Indonesia \\
\hline Sri Utami Zuliana & UIN Sunan Kalijaga & Indonesia \\
\hline
\end{tabular}




\section{4th International Conference on Information Technology, Information Systems and Electrical Engineering (ICITISEE)}

Parallel Session 1-A

Evaluation of Golomb Ruler Optimum Performance for NG-PON2 Networks

Satrio Priambodo (Telkom University, Indonesia), Brian Pamukti (Telkom University, Indonesia), Akhmad Hambali (Telkom University, Indonesia)

Entropy and Information Gain Analysis on Low Cost BCI for Motorbike Users to Prevent Accident

Rolly Maulana Awangga (Institut Teknologi Bandung \& Politeknik Pos Indonesia, Indonesia), Syafrial Fachri Pane (Applied Bachelor Program of Informatics Engineering, Politeknik Pos Indonesia, Indonesia), Dinda Majesty (Politeknik Pos Indonesia, Indonesia), Moch Yusuf Asyhari (Universitas Islam Indonesia, Indonesia)

Study on C-Band Electromagnetic Wave Absorber made of S-Ring Resonator

Farhan Fathir Lanang (Telkom University, Indonesia), Levy Olivia Nur (Telkom University, Indonesia), Budi Syihabuddin (Telkom University, Indonesia), Bambang Setia Nugroho (Telkom University, Indonesia), Agus D. Prasetyo (Telkom University, Indonesia), Heroe Wijanto (Telkom University, Indonesia)

Performance evaluation of M-ary modulated DCO-OFDM in an Indoor Visible Light Communication System

Nurul Fatma Milia (Telkom University, Indonesia), Erna Sugesti (Telkom University, Indonesia), Desti Madya Saputri (Telkom University, Indonesia), Brian Pamukti (Telkom University, Indonesia)

Impact Analysis of Location and Penetration Level of DFIG on Small Signal Stability of Power System

Avrin Nur Widiastuti (UGM, Indonesia), Sarjiya Sarjiya (Gadjah Mada University, Indonesia), Sasongko Hadi (Universitas Gadjah Mada, Indonesia)

Analysis of Transient Signal using Hilbert-Huang Transform for Chatter Monitoring in Turning Process

Agus Susanto (State Polytechnic of Madiun, Indonesia), Keiji Yamada (Hiroshima University, Japan), Ryutaro Tanaka (Hiroshima University, Japan), Muizuddin Azka (Agency for the Assessment and Application of Technology (BPPT), Indonesia), Katsuhiko Sekiya (Hiroshima University, Japan), Murman Dwi Prasetio (Telkom University, Indonesia), Putri Novia (State Polytechnic of Madiun, Indonesia)

Parallel Session 1-B

Automatic Cacao Pod Detection Under Outdoor Condition Using Computer Vision

Yulia Ekawaty (Universitas Hasanuddin, Indonesia), Indrabayu A (Hasanuddin University, Indonesia), Intan Sari Areni (Hasanuddin University, Indonesia)

Using Big Data and Al to Examine Product Engagement in Social Media Influencer Posts

Stuart Barnes (King's College London, United Kingdom (Great Britain)), Richard Rutter (Australian College of Kuwait, United Kingdom (Great Britain))

The Best Parameters to Select Instagram Account for Endorsement using Web Scraping

Muhammad Ichwandar Akrianto (Universitas Amikom Yogyakarta, Indonesia), Anggit Dwi Hartanto (Universitas Amikom Yogyakarta,

Indonesia), Adri Priadana (Universitas Jenderal Achmad Yani Yogyakarta, Indonesia)

Decision Support System for Boarding house Search Using Topsis Algorithm

Ainul Yaqin (Universitas Amikom Yogyakarta, Indonesia), Akhmad Dahlan (Universitas Amikom Yogyakarta, Indonesia), Tonny Hidayat (Universitas AMIKOM Yogyakarta, Indonesia), Reza Mardiansyah Putra (Amikom, Indonesia)

The Selection of Periodic Salary Increment of Civil Servants using Fuzzy MADM

Wahyuni Eka Sari (Politeknik Pertanian Negeri Samarinda, Indonesia), Silmi Fauziati (Universitas Gadjah Mada, Indonesia)

Time-Frequency Analysis (TFA) method for load identification on Non-Intrusive Load Monitoring

Nur Iksan (Universitas Negeri Semarang, Indonesia), Erika Devi Udayanti (Universitas Dian Nuswantoro, Indonesia)

Parallel Session 1-C

Automatically Regulates Non Player Character Behavior Using Fuzzy Logic As An Artificial Intelligence Mechanism For Action Makers Tonny Hidayat (Universitas AMIKOM Yogyakarta, Indonesia), Ika Astuti (Universitas Amikom Yogyakarta, Indonesia), Akhmad Dahlan (Universitas Amikom Yogyakarta, Indonesia)

Comparison Analysis of the Implementation of the AHP and AHP-PROMETHEE Methods for the Selection of Trainees

Dewi Anisa Istiqomah (Universitas AMIKOM Yogyakarta, Indonesia), Vikky Aprelia (Universitas AMIKOM Yogyakarta, Indonesia)

Clustering K Means for Criteria Weighting With Improvement Result of Alternative Decisions Using SAW and TOPSIS

Erna Daniati (Indonesia \& Universitas Nusantara PGRI Kediri, Indonesia), Hastari Utama (Universitas Amikom Yogyakarta, Indonesia)

Animal metamorphosis learning media using android- Based augmented reality technology

Agus Purwanto (Lecturer of University Amikom Yogyakarta \& CREATIVE DIRECTOR OF PARAMA CREATIVE, Indonesia), Mei Parwanto Kurniawan (University of AMIKOM Yogyakarta, Indonesia), Ahmad Zaid Rahman (Universitas AMIKOM Yogyakarta, Indonesia)

Augmented Reality of Android-Based Learning Media of Sun and Earth Structure

Mei Parwanto Kurniawan (University of AMIKOM Yogyakarta, Indonesia), Agus Purwanto (Lecturer of University Amikom Yogyakarta \& CREATIVE DIRECTOR OF PARAMA CREATIVE, Indonesia), Muhammad Fahmi Mansur (University of Amikom Yogyakarta, Indonesia) 
Implementation Least Means Square Algorithm for Real-Time Active Noise Cancellation on FPGA

Muhammad Nur Shahreen Osman (Universiti Teknologi MARA, Malaysia), Ili Shairah Abdul Halim (Universiti Teknologi MARA \& Faculty of

Electrical Engineering, Malaysia), Siti Lailatul Mohd Hassan (Universiti Teknologi Mara, Malaysia), A'zraa Afhzan Ab Rahim (Universiti

Teknologi MARA, Malaysia), Noor Ezan Abdullah (Universiti Teknologi Mara, Malaysia)

\section{Parallel Session 1-D}

Comparison of Pornographic Image Classification based on Texture, Color, and Shape Features

I Wayan Pandu Swardiana (Universitas AMIKOM Yogyakarta, Indonesia), Arief Setyanto (Universitas AMIKOM Yogyakarta, Indonesia), Sudarmawan Sudarmawan (AMIKOM Yogyakarta University, Indonesia)

Implementation of 2DPCA and SOM Algorithms to Determine Sex According to Lip Shapes

Nor Hikmah (Universitas AMIKOM, Indonesia)

Comparison of Scale Invariant Feature Transform and Speed Up Robust Feature for Image Forgery Detection Copy Move

Reflan Nuari (Amikom University Yogyakarta, Indonesia), Ema Utami (Universitas Amikom Yogyakarta, Indonesia), Suwanto Raharjo (Informatics of The Institut Sains \& Teknologi AKPRIND Yogyakarta, Indonesia)

Potential Detection of Lentigo Maligna Melanoma on Solar Lentigines Image Based on Android

Casi Setianingsih (Telkom University, Indonesia)

Personality Features Identification from Handwriting Using Convolutional Neural Networks

Sri Fatimah (Universitas Jenderal Achmad Yani, Indonesia), Esmeralda Contessa Djamal (Universitas Jenderal Achmad Yani, Indonesia),

Faiza Renaldi (Universitas Jenderal Achmad Yani, Indonesia)

Comparison of Naive Bayes and K-NN method on Tuition Fee Payment Overdue Prediction

Kusrini Kusrini (AMIKOM Yogyakarta University, Indonesia), Emha Taufiq Luthfi (Universitas AMIKOM Yogyakarta, Indonesia), Muqorobin

Muqorobin (Universitas AMIKOM Yogyakarta, Indonesia), Robi Abdullah (Universitas AMIKOM Yogyakarta, Indonesia)

\section{Parallel Session 1-E}

Impact of Device Orientation for Visible Light Communication in Closed Room

Amirullah Wijayanto (Telkom University, Indonesia), Kris Sujatmoko (Telkom University, Indonesia), Brian Pamukti (Telkom University, Indonesia)

Evaluate Number of LED on Reflector Room for Optical Wireless Communication

Dyndra Ramadhanti (Telkom University, Indonesia), Brian Pamukti (Telkom University, Indonesia), Kris Sujatmoko (Telkom University, Indonesia)

Blockchain-based Secure Data Storage for Door Lock System

Ulfah Nadiya (Indonesian Institute of Science, Indonesia), Muhammad Ilham Rizqyawan (Indonesian Institute of Sciences, Indonesia), Oka Mahendra (Indonesian Institute of Sciences, Indonesia)

Detection System for Cigarette Smoke

Junaidy B Sanger (Universitas Katolik De La Salle Manado, Indonesia), Lanny Sitanayah (Universitas Katolik De La Salle Manado, Indonesia), Vivie D. Kumenap (Universitas Katolik De La Salle Manado, Indonesia)

A Novel Approach to Resource Starvation Attacks on MQTT Brokers

Ricardo Da Paz (Edith Cowan University, Australia), Aiden Sehovic (Edith Cowan University, Australia), David Cook (Edith Cowan University, Australia), Leisa J Armstrong (Edith Cowan University, Australia)

Design and Implementation of Node Gateway with MQTT and CoAP Protocol for IoT Applications

Ahmad Zainudin (Politeknik Elektronika Negeri Surabaya, Indonesia), Mohamad Fahmi Syaifudin (PENS, Indonesia), Nanang Syahroni (Politeknik Elektronika Negeri Surabaya, Indonesia)

Parallel Session 2-A

Electroencephalograph Recording with Ten-Twenty Electrode System Based on Arduino Mega 2560 Titis Bagus Kurnianadi (Soegijapranata Catholic University, Indonesia), Florentinus Budi Setiawan (Soegijapranata Catholic University, Indonesia)

Diminish the Peak Value of the Cogging Torque by Modifying of the Stator Teeth Tip Geometry

Herlina Wahab (Sriwijaya University \& University of Indonesia, Indonesia), Rudy Setiabudy (Universitas Indonesia (UI), Indonesia), Syamsuri Zaini (Sriwijaya University, Indonesia)

Level Crossing Rate Impact on Routing Performance in Adhoc Networks for Device-to-Device Communication

Istikmal Istikmal (Telkom University, Indonesia), Edwar Edwar (Telkom University, Indonesia)

An Adaptive Scaling Factor for Multiple Watermarking Scheme

Dhani Ariatmanto (Universitas Amikom Yogyakarta, Indonesia), Ferda Ernawan (Universiti Malaysia Pahang, Malaysia) 
Development of Internet-of-Things based Building Monitoring System for Supporting the Disaster Mitigation in The City

Asep Najmurrokhman (Universitas Jenderal Achmad Yani, Indonesia), Kusnandar Kusnandar, k (UNJANI \& UNJANI, Indonesia), Udin Komarudin (Universitas Jenderal Achmad Yani, Indonesia), Ahmad Daelami (Universitas Jenderal Achmad Yani, Indonesia), Restu Arisandy (Universitas Jenderal Achmad Yani, Indonesia)

MQTT Performance as a Message Protocol in an loT based Chili Crops Greenhouse Prototyping

Dania Eridani (Diponegoro University, Indonesia), Kurniawan Martono (Diponegoro University, Indonesia)

Development of Automatic Waste Segregator with Monitoring System

A'zraa Afhzan Ab Rahim (Universiti Teknologi MARA, Malaysia), Nurisha Hania Kamarudin (UiTM, Malaysia), Noor Ezan Abdullah (Universiti Teknologi Mara, Malaysia), Ili Shairah Abdul Halim (Universiti Teknologi MARA \& Faculty of Electrical Engineering, Malaysia), Siti Lailatul Mohd Hassan (Universiti Teknologi Mara, Malaysia)

Quantization Effect on 5 G Millimeter Wave Communication

Nasrullah Armi (Indonesian Institute of Sciences \& Telkom University, Indonesia), Chaeriah Bin Ali Wael (Indonesian Institute of Sciences, Indonesia), Arumjeni Mitayani (Indonesian Institute of Sciences, Indonesia), Arief Suryadi Satyawan (Waseda University, Japan \& Indonesian Institute of Sciences, Indonesia), Galih Nugraha Nurkahfi (Indonesian Institute of Science(LIPI), Indonesia)

\section{Parallel Session 2-B}

Human Perception Evaluation toward End of File Steganography Method's Implementation Using Multimedia File (Image, Audio, and Video) Rini Indrayani (Universitas Amikom Yogyakarta, Indonesia)

Hybrid Encryption Technique using Cyclic Bit Shift and RC4

Dina Evita Sari (Dian Nuswantoro University, Indonesia), Happy Niti Noor Muchsin (Dian Nuswantoro University, Indonesia), De Rosal Ignatius Moses Setiadi (Dian Nuswantoro University, Indonesia), Christy Atika Sari (Dian Nuswantoro University, Indonesia), Eko Hari Rachmawanto (Dian Nuswantoro University, Indonesia)

Steganographic-Algorithm and Length Estimation Classification on MP3 Steganalysis with Convolutional Neural Network Muhammad Rizki Duwinanto (Bandung Institute of Technology, Indonesia), Rinaldi Munir (Institut Teknologi Bandung, Indonesia)

QIM-based Audio Watermarking using Polar-based Singular Value in DCT Domain

Gelar Budiman (Telkom University \& Institute Technology Bandung, Indonesia), Ledya Novamizanti (Telkom University, Indonesia), Allwinnaldo Allwinnaldo (Telkom University, Indonesia)

Dual Encryption Method for File Security

Daniel Mahardika Yusuf (Dian Nuswantoro University, Indonesia), De Rosal Ignatius Moses Setiadi (Dian Nuswantoro University, Indonesia) Christy Atika Sari (Dian Nuswantoro University, Indonesia), Eko Hari Rachmawanto (Dian Nuswantoro University, Indonesia), Rabei Raad Al (Universiti Tun Hussein Onn Malaysia, Malaysia)

Design of Blockchain-Based Electronic Election System Using Hyperledger: Case of Indonesia

Donny Seftyanto (Badan Siber dan Sandi Negara, Indonesia), Amiruddin Amiruddin (Sekolah Tinggi Sandi Negara \& Badan Siber dan Sandi Negara, Indonesia), Arif Rahman Hakim (Sekolah Tinggi Sandi Negara, Indonesia)

Mitigation of Cryptojacking Attacks Using Taint Analysis

Arief Dwi Yulianto (Telkom University, Indonesia), Muhammad Al Makky (Telkom University, Indonesia), Parman Sukarno (Telkom University, Indonesia), Aulia Arif Wardana (Telkom University, Indonesia)

User Satisfaction Levels Sentiment Analysis Toward Goods Delivery Service On Twitter Using Support Vector Machine Algorithm (SVM)

Andia Enggar Mayasari (Universitas AMIKOM Yogyakarta, Indonesia), Anggit Dwi Hartanto (Universitas Amikom Yogyakarta, Indonesia)

Parallel Session 2-C

Planar Dipole MIMO Array Antenna for Mobile Robot Communications at $5.6 \mathrm{GHz}$

Muhsin Muhsin (Institut Teknologi Telkom Surabaya, Indonesia)

Natural Disaster Application on Big Data and Machine Learning: A Review

Rania Arinta (Universitas Atma Jaya Yogyakarta, Indonesia), Andi Wahju Rahardjo Emanuel (Universitas Atma Jaya Yogyakarta, Indonesia)

Machine Learning Classifiers for Autism Spectrum Disorder: Review

Dadang Eman (Universitas Atma Jaya Yogyakarta, Indonesia), Andi Wahju Rahardjo Emanuel (Universitas Atma Jaya Yogyakarta, Indonesia)

Comparison of Classification Methods using Historical Loan Application Data

Yohanes R. Laberto Kelen (Universitas Atma Jaya Yogyakarta, Indonesia), Andi Wahju Rahardjo Emanuel (Universitas Atma Jaya Yogyakarta, Indonesia)

The Undersampling Effects on RANDSHUFF Oversampling Algorithms

Tora Fahrudin (University of Telkom, Indonesia)

Meta-Algorithms for Improving Classification Performance in the Web-phishing Detection Process

Anggit Ferdita Nugraha (Universitas AMIKOM Yogyakarta, Indonesia), Luthfia Rahman (Universitas Gadjah Mada, Indonesia)

The Prototype of Decision Support System For Selecting The Lands of Crops

Dema Mathias Lumban Tobing (Universitas Amikom Yogyakarta, Indonesia), Julia Kurniasih (Amikom University, Indonesia), Yulius Nahak Tetik (Amikom University, Indonesia), Kusrini Kusrini (AMIKOM Yogyakarta University, Indonesia) 
Comparison Of SIFT and SURF Methods For Porn Image Detection

Hartatik Hartatik (STMIK AMIKOM Yogyakarta, Indonesia), Arief Setyanto (Universitas AMIKOM Yogyakarta, Indonesia), Kusrini Kusrini

(AMIKOM Yogyakarta University, Indonesia), I Made Artha Agastya (Universitas Amikom Yogyakarta, Indonesia)

Parallel Session 2-D

Self-Organizing Map (SOM) For Diagnosis Coronary Heart Disease

Triyanna Widiyaningtyas (Universitas Negeri Malang, Indonesia), Ilham A. E. Zaeni (State University of Malang, Indonesia), Putri Wahyuningrum (Universitas Negeri Malang, Indonesia)

Effect of Giving N Value on ADASYN-N Method for Classification of Imbalanced Nominal Data

Sri Rahayu (Universitas Janabadra, Indonesia), Jeffry Andhika Putra (Universitas Janabadra, Indonesia), Yumarlin MZ (Universitas Janabadra, Indonesia)

Sentiment Analysis in Airline Tweets Using Mutual Information for Feature Selection

Hastari Utama (Universitas Amikom Yogyakarta, Indonesia)

Optimization Of Parameter Support Vector Machine (SVM) using Genetic Algorithm to Review GO-JEK Services

Windha Mega Pradnya Dhuhita (Universitas AMIKOM Yogyakarta, Indonesia), Haryoko Haryoko (University of AMIKOM Yogyakarta,

Indonesia)

A Review of Long Short-Term Memory Method for Hate Speech Classification on Twitter

Syahrul Syafaat Syam (Telkom University, Indonesia), Budhi Irawan (Telkom University, Indonesia), Casi Setianingsih (Telkom University, Indonesia)

Comparison Performance of Decision Tree Classification Model for Spam Filtering with or without the Recursive Feature Elimination (RFE) Approach

Ahmad Fikri Zulfikar (University of Pamulang \& Universiry of Bina Nusantara, Indonesia)

Comparison of Modulation Schemes toward Coverage Area in indoor Visible Light Communication

Andrik Supadiyanto (Telkom University, Indonesia), Brian Pamukti (Telkom University, Indonesia), Desti Madya Saputri (Telkom University, Indonesia), Nur Andini (Telkom University, Indonesia)

Parallel Session 2-E

Speech recognition for Indonesian language and its application to home automation

Zulkarnaen Hatala (Politeknik Negeri Ambon, Indonesia)

Aspect and Opinion Word Extraction on Opinion Sentences in Bahasa Indonesia using Rule Based Generated from Regular Expression Yuliana Setiowati (Politeknik Elektronika Negeri Surabaya, Indonesia), Fitri Setyorini (Politeknik Elektronika Negeri Surabaya, Indonesia), Afrida Helen (Universitas Padjadjaran (Unpad), Indonesia)

The Use Matriks of Linear and Quadratic Regression to Predict Number Electricity Distributed in Indonesia

Desty Rakhmawati (Universitas Amikom Purwokerto, Indonesia), Hendra Marcos (STMIK AMIKOM Purwokerto, Indonesia), Utami Puspita (Universitas AMIKOM Purwokerto, Indonesia), Uswatun Hasanah (STMIK Amikom Purwokerto, Indonesia)

An Optimization of a Lexicon Based Sentiment Analysis Method on Indonesian App Review

Bayu Trisna Pratama (Universitas Amikom Yogyakarta, Indonesia), Ema Utami (Universitas Amikom Yogyakarta, Indonesia), Andi Sunyoto (Universitas AMIKOM Yogyakarta, Indonesia)

Classification of Citation Sentence for Filtering Scientific References

Ghoziyah Haitan Rachman (Institut Teknologi Bandung, Indonesia), Masayu Leylia Khodra (Institut Teknologi Bandung, Indonesia), Dwi H Widyantoro (Institut Teknologi Bandung, Indonesia)

Indonesian Part of Speech Tagging Using Hidden Markov Model - Ngram \& Viterbi

Denis Eka Cahyani (Universitas Sebelas Maret, Indonesia)

Mining Student Feedback to Improve the Quality of Higher Education through Multi Label Classification, Sentiment Analysis, and Trend Topic Calandra Alencia Haryani (Universitas Indonesia \& Universitas Pelita Harapan, Indonesia), Achmad Hidayanto (University of Indonesia, Indonesia), Nur Fitriah Ayuning Budi (Universitas Indonesia, Indonesia), Zaenal Abidin (Universitas Negeri Semarang, Indonesia), Theresia Wati (Universitas Pembangunan Nasional Veteran Jakarta, Indonesia)

Mobile Business Intelligence Assistant ( $m$-BELA) for Higher Education Executives

Mohamad Irwan Afandi (Universitas Pembangunan Nasional Veteran Jawa Timur, Indonesia), Eka Dyar Wahyuni (Universitas Pembangunan Nasional Veteran Jawa Timur, Indonesia), Siti Mukaromah (Universitas Pembangunan Nasional Veteran Jawa Timur, Indonesia)

Parallel Session 3-A

Design of PID Controllers for Speed Control of Three Phase Induction Motor Based on Direct-Axis Current (Id) Coordinate Using IFOC Indra Ferdiansyah (Politeknik Elektronika Negeri Surabaya, Indonesia), Diah Yanaratri (Politeknik Elektronika Negeri Surabaya, Indonesia), Lucky Pradigta Setiya Raharja (Politeknik Elektronika Negeri Surabaya, Indonesia), Era Purwanto (Electronic Engineering Polytechnic Institute Of Surabaya, Indonesia) 
Dynamic Source Routing and Optimized Link State Routing Performance in Multipath Fading Environment with Dynamic Network Topology Istikmal Istikmal (Telkom University, Indonesia), Agus Subekti (Indonesian Institute of Sciences, Indonesia), Doan Perdana (Telkom University, Indonesia), Ridha Negara (Telkom University, Indonesia), Arif Indra Irawan (Telkom University, Indonesia), Sussi Sussi (Telkom University, Indonesia)

Comparative Analysis of Codec G.729 and G.711 on IEEE 802.11AH with MCS and Raw Slot Change Mechanism for VOIP Service Doan Perdana (Telkom University, Indonesia), Istikmal Istikmal (Telkom University, Indonesia)

Flooding Detection System Based on Water Monitoring and ZigBee Mesh Protocol

Herman Yuliandoko (State Polytehnic of Banyuwangi, Indonesia), Abdul Rohman (State Polytechnic of Banyuwangi, Indonesia)

Performance Comparison of Blackman, Bartlett, Hanning, and Kaiser Window for Radar Digital Signal Processing

Sulis Tyaningsih (Indonesian Institute of Science (LIPI), Indonesia), Prasetyo Putranto (Indonesian Institute of Sciences, Indonesia),

Taufiqqurrachman Taufiq (National Taiwan University of Science and Technology \& Indonesian Institute of Sciences, Indonesia), Winy Desvasari (Indonesian Institute of Sciences, Indonesia), Yusuf Nur Wijayanto (Indonesian Institute of Sciences (LIPI), Indonesia),

Pamungkas Daud (Indonesian Institute of Sciences, Indonesia), Dadin Mahmudin (Indonesian Institute of Sciences (LIPI), Indonesia), Deni

Permana Kurniadi (LIPI, Indonesia), Arief Rahman (Indonesian Institute of Sciences, Indonesia), Sri Hardiati (Indonesian Institute of Science (LIPI), Indonesia), Arie Setiawan (Indonesian Institute of Sciences, Indonesia), Fajri Darwis (Indonesian Institute of Sciences (LIPI),

Indonesia), Eko Pristianto (Indonesian Institute of Sciences, Indonesia)

Implementation of Algorithm Rabin-Karp for Thematic Determination of Thesis

Ainul Yaqin (Universitas Amikom Yogyakarta, Indonesia), Akhmad Dahlan (Universitas Amikom Yogyakarta, Indonesia), Reno Diandika Hermawan (University of Amikom, Indonesia)

Hybrid Resampling for Imbalanced Class Handling on Web Phishing Classification Dataset

Yoga Pristyanto (Universitas Amikom Yogyakarta, Indonesia), Akhmad Dahlan (Universitas Amikom Yogyakarta, Indonesia)

Parallel Session 3-B

Modeling of Time Series Data Prediction using Fruit Fly Optimization Algorithm and Triple Exponential Smoothing

Ryan Putranda Kristianto (Universitas Amikom Yogyakarta, Indonesia)

A Novel Ant Colony Optimization Algorithm for Waste Collection Problem

Sarifah Putri Raflesia (Universitas Sriwijaya \& Institut Teknologi Bandung, Indonesia), Anugrah K Pamosoaji (Universitas Atma Jaya

Yogyakarta, Indonesia)

Fuzzy Hierarchical Model and Particle Swarm Optimization in Gas Leakage Detector Mobile Robot

Kanda Januar Miraswan (Universitas Sriwijaya, Indonesia), Muhammad Ali Buchari (Universitas Sriwijaya, Indonesia), Rizki Kurniati

(Universitas Sriwijaya, Indonesia)

Optimization of Weight Backpropagation with Particle Swarm Optimization for Student Dropout Prediction

Eka Yulia Sari (Universitas Amikom Yogyakarta, Indonesia), Kusrini Kusrini (AMIKOM Yogyakarta University, Indonesia), Andi Sunyoto (Universitas AMIKOM Yogyakarta, Indonesia)

The Mapping of Lighting Intensity from the Light Distribution on LED and CFL Lamps

Herlina Wahab (Sriwijaya University \& University of Indonesia, Indonesia), Rudy Setiabudy (Universitas Indonesia (UI), Indonesia)

Impact of STATCOM Installation on Power System's Voltage Stability Performance

Nurriza Kholifatulloh Hasanah (Universitas Gadjah Mada, Indonesia), Lesnanto Multa Putranto (UGM, Indonesia), Sasongko Hadi (Universitas Gadjah Mada, Indonesia), Febian Melwa Reksa Aditya (Universitas Gadjah Mada, Indonesia)

Analysis Of Overclock Ram Galax Hall Of Fame For Daily Needs

Rizqi Sukma Kharisma (Universitas AMIKOM Yogyakarta, Indonesia), Miko Kastomo Putro (Universitas AMIKOM Yogyakarta, Indonesia)

\section{Parallel Session 3-C}

Analysis of Review And Rating on Consumer Trust in Jakarta Taking Online Booking Queue Based on Tam Hendrico Andre (Bina Nusantara University, Indonesia), Sfenrianto Sfenrianto (Bina Nusantara University, Indonesia), Gunawan Wang (Bina Nusantara University, Indonesia), Pangondian Prederikus (Bina Nusantara, Indonesia)

Analysis of the Effect of Security and Trust on Buying Decision On the Tokopedia Mobile Apps

Edward Chandra (Bina Nusantara University, Indonesia), Stefanie Liu (Bina Nusantara University, Indonesia), Sfenrianto Sfenrianto (Bina Nusantara University, Indonesia), Gunawan Wang (Bina Nusantara University, Indonesia)

Behavior Intention of Information Technology Students in Using Youtube as Learning Resources

Paul Weniko (Bina Nusantara University, Indonesia), Gunawan Wang (Bina Nusantara University, Indonesia), Sfenrianto Sfenrianto (Bina Nusantara University, Indonesia), Muhammad Aldenny (Bina Nusantara University, Indonesia)

Analysis of the Effect of Trust on Purchase Intention in E-Commerce Integration for Vendors \& Event Organizer

Dina Ikramina (Bina Nusantara University, Indonesia), Sfenrianto Sfenrianto (Bina Nusantara University, Indonesia), Gunawan Wang (Bina Nusantara University, Indonesia)

Evaluating the Usability of Heuristics within Telegram using the Linear Regression Method

Rumini Rumini (Universitas AMIKOM Yogyakarta, Indonesia) 
Social Network Users Switching Platforms Behaviour: A Proposal for Research Explorations using a Mixed Method Approach Timothy McBush Hiele (Ming Chuan University, Taiwan), Andree E. Widjaja (Universitas Pelita Harapan, Indonesia), Calandra A. Haryani (Universitas Pelita Harapan, Tangerang, Indonesia)

Fingerprint Presence Fraud Detection Using Tight Clustering on Employee's Presence and Activity Data

Irfan Kamil (Institut Teknologi Bandung, Indonesia), Bambang Pharmasetiawan (Institut Teknologi Bandung, Indonesia)

\section{Parallel Session 3-D}

Analysis of Dominants Game Elements using the Sillaots Parameters and Octalysis Framework on the Google Play Store

Dema Mathias Lumban Tobing (Universitas Amikom Yogyakarta, Indonesia), Emma Utami (Universitas AMIKOM Yogyakarta, Indonesia), Hanif Fatta (Universitas AMIKOM Yogyakarta, Indonesia)

Pregnancy Mapping and Monitoring Web Based Geographic's Information System

Arief Munandar (Universitas AMIKOM Yogyakarta, Indonesia), Arief Setyanto (Universitas AMIKOM Yogyakarta, Indonesia), Suwanto Raharjo (Informatics of The Institut Sains \& Teknologi AKPRIND Yogyakarta, Indonesia), Gunawan Wicahyono (Amikom University Yogyakarta, Indonesia)

Integration of K-Means Clustering and Naive Bayes Classification Algorithms for Smart AC Monitoring and Control in WSAN

Ryan Putranda Kristianto (Universitas Amikom Yogyakarta, Indonesia), Banu Santoso (Universitas AMIKOM Yogyakarta, Indonesia)

Optimization of Transmission Expansion Planning Considering the System Losses: A Case Study of the Garver's 6-Bus System Afif Amalul Arifidin (Universitas Gadjah Mada, Indonesia), Sasongko Hadi (Universitas Gadjah Mada, Indonesia), Lesnanto Multa Putranto (UGM, Indonesia), Muhammad Yasirroni (Universitas Gadjah Mada, Indonesia)

Real-Time Irradiance Estimation Based on Maximum Power Current of Photovoltaic

Moh Syaiful Imam (Politeknik Elektronika Negeri Surabaya (PENS), Indonesia), Eka Prasetyono (Politeknik Elektronika Negeri Surabaya, Indonesia), Epyk Sunarno (Politeknik Elektronika Negeri Surabaya, Indonesia)

What are Customers Really Need in Ride Hailing Applications?Signaling Electronic Service Quality via E-CRM Features

Tifanny Nabarian (Universitas Indonesia, Indonesia, Indonesia), Yudho Sucahyo (University of Indonesia, Indonesia), Arfive Gandhi (Universitas Indonesia, Indonesia), Yova Ruldeviyani (Universitas Indonesia, Indonesia)

Realization of Point Cloud Maps Using ROS \& Visual Sensor on Raspberry Pi 3 Based Mobile Robot

Husnairi Ardan Miranto (Telkom University, Indonesia), Agung Nugroho Jati (Telkom University, Indonesia), Casi Setianingsih (Telkom University, Indonesia)

Parallel Session 3-E

Essential Blockchain Technology Adoption factors in Pharmaceutical Industry

Surjandy Surjandy (Bina Nusantara University, Indonesia), Erick Fernando (Bina Nusantara University, Indonesia), Meyliana Meyliana (Bina Nusantara University, Indonesia)

Performance Evaluation of XPath Routing Protocol in Data Center Network using NS3-Simulator

Gerry Wowiling (Universitas Indonesia, Indonesia), Hermawan Rahman Sholeh (University of Indonesia, Indonesia), Ruki Harwahyu (Universitas Indonesia \& Universitas Indonesia, Indonesia), Riri Fitri Sari (University of Indonesia, Indonesia)

Job Seeker Profile Classification of Twitter Data Using the Naïve Bayes Classifier Algorithm Based on the DISC Method

Anggit Dwi Hartanto (Universitas Amikom Yogyakarta, Indonesia), Ema Utami (Universitas Amikom Yogyakarta, Indonesia), Sumarni Adi (Universitas AMIKOM Yogyakarta, Indonesia), Harish Setyo Hudnanto (Universitas AMIKOM Yogyakarta, Indonesia)

The Impact Of Features Selection On Performance Of Artificial Neural Network In Diagnosis Of Diabetic Retinopathy

Tri Astuti (Universitas Amikom Purwokerto, Indonesia), Rizki Wahyudi (STMIK Amikom Purwokerto, Indonesia), Uswatun Hasanah (STMIK Amikom Purwokerto, Indonesia), Bambang Pilu Hartato (STMIK AMIKOM Purwokerto, Indonesia), Zanuar Rifa'i (Stmik Amikom Purwokerto, Indonesia)

RS Code and Compressive Sampling on Video Watermarking-based DWT-SVD

Ledya Novamizanti (Telkom University, Indonesia)

Trending Topic Classification for Single-Label Using Multinomial Naive Bayes (MNB) and Multi-Label Using K-Nearest Neighbors (KNN) Denis Eka Cahyani (Universitas Sebelas Maret, Indonesia)

Influence of Voltage System-Level to the dimensions and performance of Squirrel Cage Induction Motor Three Phase 50Hz $5 \mathrm{HP}$ for Electric Vehicles

Danang Wijaya (UGM, Indonesia), Iftitah Imawati (Universitas Gadjah Mada, Indonesia) 


\section{General Chair}

Kusrini Kusrini (AMIKOM Yogyakarta University, Indonesia)

\section{Publication Chair}

Sumarni Adi (Universitas AMIKOM Yogyakarta, Indonesia)

Akhmad Dahlan (Universitas Amikom Yogyakarta, Indonesia)

\section{Secretary}

Yoga Pristyanto (Universitas Amikom Yogyakarta, Indonesia)

Nila Puspitasari (Universitas AMIKOM Yogyakarta, Indonesia)

\section{Treasurer}

Sumarni Adi (Universitas AMIKOM Yogyakarta, Indonesia)

\section{Chair of Technical Program Committee}

Emha Taufiq Luthfi (Universitas AMIKOM Yogyakarta, Indonesia)

Lesnanto Multa Putranto (UGM, Indonesia)

Arief Setyanto (Universitas AMIKOM Yogyakarta, Indonesia)

Andi Sunyoto (Universitas AMIKOM Yogyakarta, Indonesia)

Ferry Wahyu Wibowo (Universitas Amikom Yogyakarta, Indonesia) 


\section{Natural Disaster Application on Big Data and Machine Learning: A Review}

\author{
$1^{\text {st }}$ Rania Rizki Arinta \\ Magister Teknik Informatika \\ Universitas Atma Jaya Yogyakarta \\ Yogyakarta, Indonesia \\ raniarinta@gmail.com
}

\author{
$2^{\text {nd }}$ Andi W.R. Emanuel \\ Magister Teknik Informatika \\ Universitas Atma Jaya Yogyakarta \\ Yogyakarta, Indonesia \\ andi.emanuel@uajy.ac.id
}

\begin{abstract}
Natural disasters are events that are difficult to avoid. There are several ways of reducing the risks of natural disasters. One of them is implementing disaster reduction programs. There are already several developed countries that apply the concept of disaster reduction. In addition to disaster reduction programs, there are several ways to predict or reducing the risks using artificial intelligence technology. One of them is big data, machine learning, and deep learning. By utilizing this method at the moment, it facilitates tasks in visualizing, analyzing, and predicting natural disaster. This research will focus on conducting a review process and understanding the purpose of machine learning and big data in the area of disaster management and natural disaster. The result of this paper is providing insight and the use of big data, machine learning, and deep learning in 6 disaster management area. This 6-disaster management area includes early warning damage, damage assessment, monitoring and detection, forecasting and predicting, and post-disaster coordination, and response, and long-term risk assessment and reduction.
\end{abstract}

Keywords - natural disaster, review, big data, machine learning

\section{INTRODUCTION}

Natural disasters are events that result from natural processes that cannot be predicted. Natural disasters can also cause loss of life or damage property and economic losses. According to data provided by world health organizations from 1900 to 2018, there are around 14 million for all types of disasters [1]. Children are the most vulnerable groups who get direct impacts of disasters. Disaster victims come from various countries, and disasters have been threatening the lives of millions of children [2].

One effort to minimize disasters is to provide a program for reducing the risks of natural disasters. Disaster risk reduction is a concept of how communities reduce damage and victims affected by disasters. One example of risk reduction according to the Sendai framework [3] made by UNISDR (United Nations International Strategy for Disaster Reduction) is understanding the risk of the disaster. According to Goswami's research [4] states that the aim of disaster management is minimizing victims, can save victims promptly, evacuate people to save places, Reconstruct the damages immediately, Offer first aid instantly.

The technology today is big data, machine learning, along with deep learning. According to Hashem's research stated that big data is a set of techniques and technology that requires a new form of integration to find large hidden values derived from datasets that are complex, diverse, and of a massive scale. The advantage of using big data can determine the pattern obtained from data analysis and the creation of hidden information [5].

In this study, we will review and focus more on Disaster management phases, which focus more on the use of machine learning in the area of Disaster management phases, which consists of the data source used and the model/algorithm used. Because to find out whether the previous research solved the problem in the prediction area and early detection we must know the data source used already has 5'v characteristics, namely Velocity, Volume, Value, Variety, and Veracity. The performance level of the model made is good or not from the level of accuracy, precision, recall, and the execution time. The propose of this study to give an insight and the use of big data, machine learning, and deep learning from 6 disaster area which is early warning damage, damage assessment, monitoring and detection, forecasting and predicting, and post-disaster coordination, and response, and long-term risk assessment and reduction. But also give the data source that the previous research

\section{RELATED WORK}

Based on existing research, Manzhu Yu researches on natural disasters which are divided into several parts. One of them is categorizing the articles based on major data sources which consist of satellite imagery, aerial imagery, and videos from unmanned Detection and Ranging (LiDAR), simulation, spatial data, crowdsourcing, social media, and mobile. Disaster management phases consist of 4 main parts Mitigation/prevention, Preparedness, Response, and Recovery. But in Manzhu's research, they divided the 4 parts of the Disaster Management Phases into 6 phases, which include early warning damage, damage assessment, monitoring and detection, forecasting and predicting, and post-disaster coordination, and response, and long-term risk assessment and reduction. Based on Goswami's research, he conducted a review of natural disasters in a case study in India. This research classifies the objectives of the tasks, namely Prediction, Detection, and Disaster management strategies.

Based on this data analysis, we could determine the pattern and solve the problem. By predicting the event or maybe use it for early detection. To solve this kind of problem, we need to use not only data sources but also the type of model/ algorithm to train and test the data. Most of the review focuses on the only area on the disaster management phase and the data source from the result of Manzhu study he didn't explain about the performance on the model/algorithm in the reviews. The most important part of the early detection and 
prediction is to analyze the data source and the model they use to solve the problem.

The following table 1 is a table of previous researchers that focuses on the area topic in disaster management and natural disaster using big data and machine learning approach.

TABLE I. RELATED WORK ON THE PREVIOUS REVIEW RESEARCH PAPER

\begin{tabular}{|c|c|c|}
\hline Categories & Data source & Citation \\
\hline \begin{tabular}{ll} 
- & \multicolumn{2}{l}{ Early warning } \\
damage & \\
- & \multicolumn{2}{l}{ Damage assessment } \\
- & \multicolumn{2}{l}{ Monitoring } & and \\
detection & \\
- & $\begin{array}{l}\text { Forecasting } \\
\text { predicting }\end{array}$ \\
- & and \\
Post-disaster & \\
coordination & and \\
response & \\
Long-term & risk \\
assessment & and \\
reduction &
\end{tabular} & 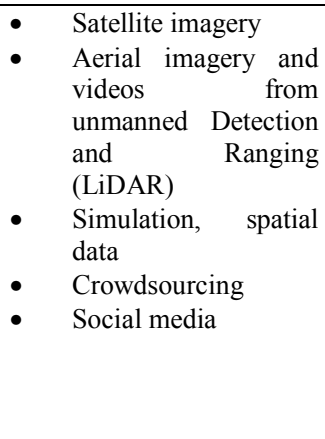 & {$[6]$} \\
\hline $\begin{array}{ll}- & \text { Prediction, } \\
\text { - } & \text { Detection } \\
\text { - } & \text { Disaster } \\
& \text { management } \\
\text { strategies }\end{array}$ & $\begin{array}{ll}\text { - } & \text { Hydrological data, } \\
\text { - } & \text { Meteorological data } \\
\text { - } & \text { twitter }\end{array}$ & {$[4]$} \\
\hline
\end{tabular}

Based on previous research such as the table above in the study, Manzhu Yu divides into 6 categories, and Goswami divides into 3 groups. Based on these categories, Manzhu's research was more detailed because the category was taken based on the concept of Disaster management phases. In this study, we will adopt 6 categories that have been created by Manzhu, and combine them with Goswami research to provide models/techniques that have been given along with the data used.

\section{METHOD}

The paper that will be chosen in the first review process is a paper that publishes on international proceedings or an article published in international journals. So from that, the article to be taken must use English. After that, the second requirement is the paper chosen is a paper published in the last five years. That is a paper published from 2014 until 2019. After that, the article was taken based on science direct[7], springer open-source [8], IEEE [9], google scholar [10], and research gate [11]. The search process for the paper by considering the abstract keyword is related to any natural disaster. The article must also discuss the fields of big data, machine learning, or deep learning. The following figure 1 explains the flow chart on how to choose the article that will be reviewed in this study.

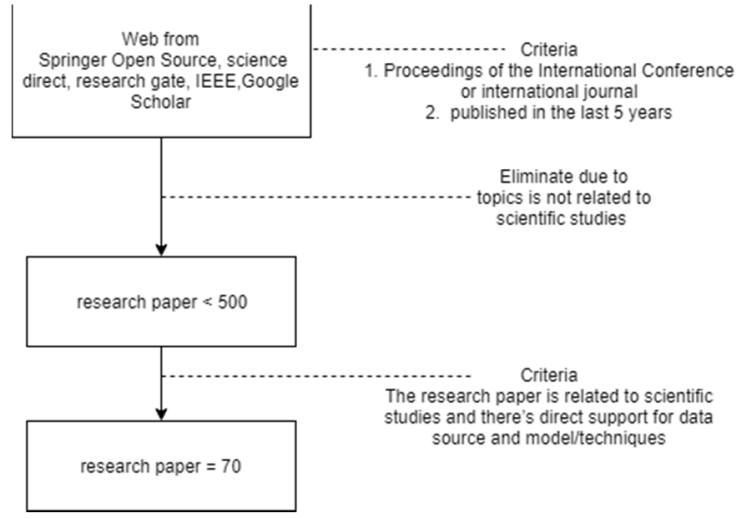

Fig. 1. Step Of Experimental Method

In figure 1 it explains the steps to getting the journal so that it is first selected based on the appropriate topic. Paper taken must be in the area of big data, machine learning, and deep learning. The chosen paper must have data source information used in the study. Along with the model/technique/algorithm used. In this paper, we will classify reviews based on frequently used data sources, often used algorithms so that it can compare and provide evaluations of datasets that are accurately used in big data research, machine learning, or deep learning. Besides that, it categorizes based on Disaster management phases that have been modified by human management, which consists of 6 categories [6]. There are 6 , namely early warning damage, damage assessment, monitoring and detection, forecasting and predicting post-disaster coordination, and response, and longterm risk assessment and reduction. Its long-term risk assessment and reduction function is for long-term disaster risk reduction. Forecasting and Predicting used to predict natural disasters that will occur. Monitoring and detection to detect or monitor disasters. Early warning Damage is used to receive relevant and timely information. Damage Assessment is an actual disaster evaluation event caused by a natural disaster event. Post-disaster recovery planning is defined as developing a set of strategies to help rebuild after a disaster occurs

\section{RESULT}

After choosing the paper that matches the criteria above, then it is to group the article based on the categories that have been made by Manzhu. After that, classify the review paper on the data source used during natural disaster research. After that, it is classifying review paper based on the model/ algorithm used in the study as with the Goswami study. In Manzhu's study, there are six categories of Disaster management phases. These categories are, among others, Long-term risk assessment and reduction, Forecasting and Predicting, Monitoring and detection, Early Warning Damage, Damage Assessment, Post-disaster Coordination, and Response. The following are the results of the paper following the six categories in Table 2.

TABLE II. DISASTER MANAGEMENT PHASES

\begin{tabular}{|l|l|}
\hline Categories & Citation \\
\hline $\begin{array}{l}\text { Long-term risk assessment and } \\
\text { reduction }\end{array}$ & {$[12],[13],[14],[15][16]$} \\
\hline Forecasting and Predicting & {$[17],[18],[19],[20],[21][22][23][2$} \\
& $4][25][26]$ \\
\hline Monitoring and detection & {$[27],[28],[29],[30],[31],[32],[33],[$} \\
& $34],[35],[36][37][38][39][40][41]$ \\
& {$[42][43][44][45][46]$} \\
\hline
\end{tabular}




\begin{tabular}{|l|l|}
\hline Early warning Damage & {$\left[\begin{array}{l}{[47,,[48],[49],[50][51][52][53][54} \\
]\end{array}\right.$} \\
\hline Damage Assessment & {$[55],[15],[56],[15],[55],[56][57][5$} \\
& $8]$ \\
\hline $\begin{array}{l}\text { Post-disaster Coordination and } \\
\text { Response }\end{array}$ & {$[59],[60][61][62][63]$} \\
\hline
\end{tabular}

Based on the results of table 2, part of the research is often done in the area of monitoring and detection. The monitoring and detection section is a component that is usually researched because based on the results of the paper to obtain data sources in the process of monitoring and detection is straightforward. Data sources that are often used are twitter data and satellite data. The data retrieval process is easy to take because it is open source data so that the research is a research that is often carried out in the area of a natural disaster.

Research that is rarely done is post-disaster coordination and response. The big data and machine learning in this area are still very uncommon. Because to conduct this area of topics, the expert has to actually examine the disaster area and carry out the infrastructure process from the damaged areas from natural disasters. Based on the results of table 2, it can be concluded that the most suitable area for disaster risk in the natural disaster area is Monitoring and detection and Forecasting and Predicting. the area is the most appropriate area to be applied in a natural disaster area because of the domain is the most suitable area to be used in the area of natural disasters because the data provided has already had 5 'v characteristics, namely Velocity, Volume, Value, Variety, and Veracity.

After categorizing according to the 6 categories, then classify based on data sources used in research on big data, machine learning, and deep learning. The following data source is used for research in table 3 .

TABLE III. TABLE DATA SOURCE FOR THE DISASTER AREA

\begin{tabular}{|l|l|}
\hline Data source & Citation \\
\hline Satellite imagery & {$[35][30][64][32][28][42][26][45]$} \\
\hline Social media & {$[47][65][66][56][55][15][67][21][$} \\
& $49]$ \\
& {$[31][36][37][16][57][23][61][52][$} \\
& $41][62][53][46][54][63]$ \\
\hline Crowdsourcing data & {$[65][17][21][20][29][16][22][39][$} \\
& $58][24][25][44]$ \\
\hline Aerial imagery & {$[59][38]$} \\
\hline Online News report & {$[51][43]$} \\
\hline
\end{tabular}

In table 3 , there is a data source used by previous research. The data sources are satellite imagery, social media, crowdsourcing data, and aerial imagery. In the above results, the data source that is often used is social media, especially Twitter. For data sources that use Twitter more, they use analysis sentiment, as Ghazaleh's research uses data source social media for sentiment analysis [68]. Zahra's research uses twitter data sources such as Ghazaleh's research for analysis sentiment [60]. On crowdsourcing data that is often taken is data provided by USGS (United States Geological Survey). One paper that uses these data is Mendoza's research [21] and Resch's research [15]. The satellite image is also used as a data source. The satellite image came from NASA like the Gokaraju study [30]. Besides that, there is satellite imagery that uses SAR (Synthetic Aperture Radar) to obtain data sources such as Wieland's research [32]. Base on the results in table 3 concluded that the data source that is often used is social media data, especially Twitter. social media data, for example, twitter to access data on tweets is accessible. Based on Nguyen's research, he uses steaming API for extracting the tweets data with a keyword "quake," "tsunami," and "earthquake." The study also explains that they could download $18 \mathrm{gb}$ twitter bases on "quake," "tsunami," and "earthquake" keyword from September 11 to December 25, 2016 [36]. Most of the satellite data, crowdsourcing, and areal image took at least two years to generate the data to understand and predict the pattern from the image. For example, in Thibaut's research, he uses data crowdsourcing from the Oklahoma Geological Survey (OGS), which only generate data 2021 seismic events from 2014 to 2016 [29]. We could conclude that social media data which is twitter data is easy to extract with the help of streaming API, not only it's easy but the volume of data is enormous we could get $18 \mathrm{gb}$ data on a tweet with a small period of time which in this case it's 4 month rather using crowdsourcing data which take approximately 2 years. After classifying the paper based on the data source, then classify the article based on the model / technique / algorithm used in the study. There are several types of models used. Table 4 discusses the model/algorithm used for the disaster area.

TABLE IV. MODEL / TECHNIQUES USED

\begin{tabular}{|l|l|}
\hline Model/techniques & Citation \\
\hline $\begin{array}{l}\text { SVSA (Support Vector Selection } \\
\text { Adaptation) }\end{array}$ & {$[28]$} \\
\hline Decision Tree & {$[34][63]$} \\
\hline Random Forest & {$[35][65][69][34][13][61]$} \\
\hline Support Vector Machine & {$[65][67][32][49][28]$} \\
& {$[34],[13][16][39][58][51][61][42]$} \\
& {$[25][62][26]$} \\
\hline Boosted Regression Trees (BRT) & {$[35]$} \\
\hline Bayesian Networks & {$[14]$} \\
\hline K-Means & {$[12][39]$} \\
\hline Naïve Bayes & {$[51][61][43]$} \\
\hline Naïve Bayes Classifiers & {$[66][69][49][57]$} \\
\hline CNN (Convolutional Neural & {$[55][36][29][38][44][45][54]$} \\
Network) & \\
\hline Latent Dirichlet Allocation (LDA) & {$[15][37][61][41]$} \\
\hline ANN (artificial neural networks) & {$[27][17][20][70][22][42]$} \\
\hline C4.5 & {$[51][63]$} \\
\hline Neural Clouds & {$[40]$} \\
\hline Logistic Regression & {$[42]$} \\
\hline
\end{tabular}

Based on the results of table 4, there are several types of algorithms used. For the algorithm, Support Vector Machine is used in research to detect changes in single- and multitemporal X-and L-bands using SAR (Synthetic Aperture Radar) image [32]. Besides that, it is also used in the research of earthquake image classification process. In this study, using SVM to compare whether the SVSA model (Support Vector Selection Adaptation) can provide increased performance at the computational time, along with the new model does not require kernels [28]. Also, the model that is often used is ANN (artificial neural network). The study used ANN for earthquake early detection, also known as precursor [27]. Also, ANN was also used to predict earthquakes magnitude in Tokyo [20]. Base on the result, we conclude that CNN works best for earthquake detection. Not only for the accuracy, but recall, precision, and the execution time is much faster. But the downside of this algorithm/model you 
need a lot of data. For example, Perol's research uses CNN for earthquake detection. He compares it with the previous study and that his model execution time only took 1 minute 1 second then the other 2 models, which took 9 days 13 hours and 48 hours to execute [29]. Not only in Perol's research but also Nguyen uses CNN for earthquake detection perform better not only in the accuracy, but also recall, pre, and score. They compare the CNN model with SVM. The reason why CNN performs better than SVM it's because of the amount of data they use. Base on that research, they use $18 \mathrm{gb}$ tweets of data. SVM performs poorly due to the amount of data. SVM works really well on smaller data rather than big amounts of data. They use the CNN model to avoid underfitting and overfitting problem [36]. The neural cloud is an algorithm that uses the combination of an Advanced K-Means (AKM) clustering algorithm and an extended Radial Basis Functions (RBF). The neural cloud is a cloud detection algorithm. Research by Pyayt et al. uses the algorithm for monitoring and flood protection [40].

Based on the result of table 2 we could see the result from figure 2 .

\section{6 disaster area response}

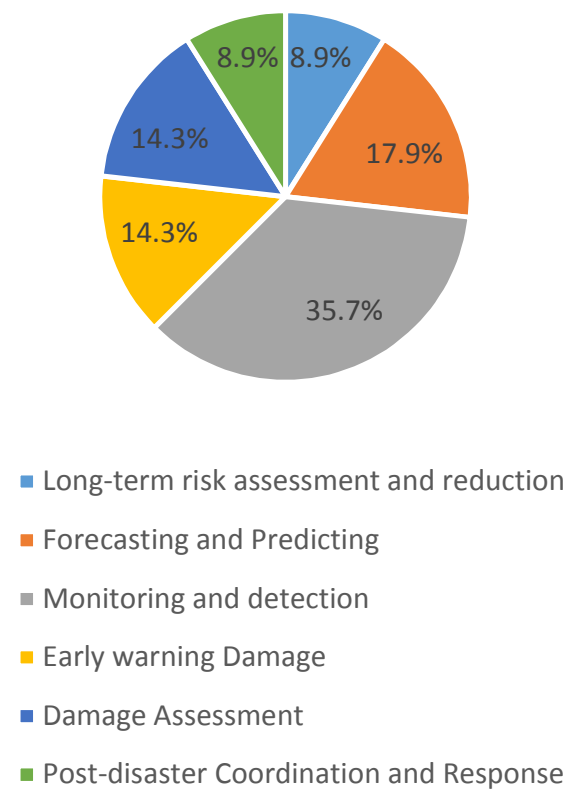

Fig. 2. Result Chart

Figure 2 explains the most common research and the most uncommon research regarding the 6-disaster area. From the result we conclude that for the 6-disaster area the most common area was monitoring and detection. The reason why this area is so common because monitoring a disaster has a big impact on the disaster management phase because if we could monitor the activities of the natural disaster, we could recognize the pattern and eventually we could predict the cause of the natural disaster. From the data source area, the most common research was social media data. The reason why it was so common because the data extraction only needed a twitter API and the data that the twitter produces up to $18 \mathrm{~GB}$ of twitter data. The most important part of big data and machine learning was to extract a large amount of data that has 5'v characteristics, namely Velocity, Volume, Value, Variety, and Veracity.

Base on the result of tables 2,3, and 4 we could conclude that big data and machine learning could help overcome the disaster management area which includes early warning damage, damage assessment, monitoring and detection, forecasting and predicting post-disaster coordination, and response, and long-term risk assessment and reduction. From this area, we could choose the right time of data source and model which one performs better for the set of problems that we choose.

\section{CONCLUSION}

Based on previous results, it can be concluded that with big data, machine learning, or deep learning can help in 6 areas of Disaster management. These areas are early warning damage, damage assessment, monitoring and detection, forecasting and predicting post-disaster coordination, and response, and long-term risk assessment and reduction. By classifying based on data sources and models/algorithms that are often used by previous researchers can help researchers who will research in the field of a natural disaster from the result we can conclude that the most common area that utilizes big data and machine learning Monitoring and detection. Monitoring and detection is the most common research in the 6 disaster management area due to the increase of data collection. The data collection on monitoring and detection is easy to extract with twitter data. And the amount of data that had been extracted with twitter API was $18 \mathrm{gb}$ twitter data. And the data has 5 'v characteristics, namely Velocity, Volume, Value, Variety, and Veracity. For the future, we will examine the model/ algorithm not only base on the level of accuracy, precision, recall, and the execution time but also the time needed to build the model itself.

\section{REFERENCES}

[1] "Number of reported disasters by type." [Online]. Available: https://ourworldindata.org/natural-disasters.

[2] Tuswadi and T. Hayashi, "Disaster Prevention Education in Merapi Volcano Area Primary Schools: Focusing on Students' Perception and Teachers' Performance," Procedia Environ. Sci., vol. 20, pp. 668-677, 2014.

[3] "2015 43291 Sendaiframeworkfordrren Disaster Reducton 2015-2030," 2015.

[4] S. Goswami, S. Chakraborty, S. Ghosh, A. Chakrabarti, and B. Chakraborty, "A review on application of data mining techniques to combat natural disasters," Ain Shams Eng. J., vol. 9, no. 3, pp. 365-378, 2018.

[5] I. A. T. Hashem, I. Yaqoob, N. B. Anuar, S. Mokhtar, A. Gani, and S. Ullah Khan, "The rise of 'big data' on cloud computing: Review and open research issues," Inf. Syst., vol. 47, pp. 98-115, 2015.

[6] M. Yu, C. Yang, and Y. Li, "Big Data in Natural Disaster Management: A Review," Geosciences, vol. 8, no. 5, p. 165, 2018.

[7] P. Sciences, "science direct," 2018. [Online]. Available: https://www.sciencedirect.com/.

[8] Springer, “springeropen," Technolo, 2018. [Online]. Available: https://www.springeropen.com/journals.

[9] IEEE, "IEEE," 2018. [Online]. Available: http://ieeexplore.ieee.org.

[10] Google Scholar, "Google Scholar," 2018. [Online]. Available: https://scholar.google.com/intl/en/scholar/about.html.

[11] L. Connect, "ResearchGate," 2018. [Online]. Available: https://www.researchgate.net/.

[12] B. Supriyadi, A. P. Windarto, T. Soemartono, and . M., "Classification of Natural Disaster Prone Areas in Indonesia using 
K-Means," Int. J. Grid Distrib. Comput., vol. 11, no. 8, pp. 87-98, 2018.

[13] C. Geiß et al., "Estimation of seismic building structural types using multi-sensor remote sensing and machine learning techniques," ISPRS J. Photogramm. Remote Sens., vol. 104, pp. 175-188, 2015

[14] J. Wang, X. Gu, and T. Huang, "Using Bayesian networks in analyzing powerful earthquake disaster chains," Nat. Hazards, vol. 68 , no. 2, pp. 509-527, 2013.

[15] B. Resch, F. Usländer, and C. Havas, "Combining machinelearning topic models and spatiotemporal analysis of social media data for disaster footprint and damage assessment," Cartogr. Geogr. Inf. Sci., vol. 45, no. 4, pp. 362-376, 2018.

[16] R. Kiatpanont, U. Tanlamai, and P. Chongstitvatana, "Extraction of actionable information from crowdsourced disaster data," $J$. Emerg. Manag., vol. 14, no. 6, pp. 377-390, 2016.

[17] K. M. Asim, F. Martínez-Álvarez, A. Basit, and T. Iqbal, "Earthquake magnitude prediction in Hindukush region using machine learning techniques," Nat. Hazards, vol. 85, no. 1, pp. 471-486, 2017.

[18] N. D. Hoang and D. T. Bui, "Predicting earthquake-induced soil liquefaction based on a hybridization of kernel Fisher discriminant analysis and a least squares support vector machine: a multidataset study," Bull. Eng. Geol. Environ., vol. 77, no. 1, pp. 191204, 2018.

[19] K. M. Asim, A. Idris, T. Iqbal, and F. Martínez-Álvarez, "Earthquake prediction model using support vector regressor and hybrid neural networks," PLoS One, vol. 13, no. 7, pp. 1-22, 2018.

[20] G. Asencio-Cortés, F. Martínez-Álvarez, A. Troncoso, and A. Morales-Esteban, "Medium-large earthquake magnitude prediction in Tokyo with artificial neural networks," Neural Comput. Appl., vol. 28, no. 5, pp. 1043-1055, 2017.

[21] M. Mendoza, B. Poblete, and I. Valderrama, "Nowcasting earthquake damages with Twitter," EPJ Data Sci., vol. 8, no. 1, 2019.

[22] F. Martínez-Álvarez, A. Troncoso, H. Quintián, and E. Corchado, "Rainfall prediction: A Deep Learning approach," Lect. Notes Comput. Sci. (including Subser. Lect. Notes Artif. Intell. Lect. Notes Bioinformatics), vol. 9648, no. June, 2016.

[23] O. Aulov and M. Halem, "Human sensor networks for improved modeling of natural disasters," Proc. IEEE, vol. 100, no. 10, pp. 2812-2823, 2012.

[24] A. Baldominos, E. Albacete, Y. Saez, and P. Isasi, "A scalable machine learning online service for big data real-time analysis," IEEE SSCI 2014 - 2014 IEEE Symp. Ser. Comput. Intell. - CIBD 20142014 IEEE Symp. Comput. Intell. Big Data, Proc., pp. 1-8, 2015.

[25] X. Yu, "Disaster prediction model based on support vector machine for regression and improved differential evolution," Nat. Hazards, vol. 85, no. 2, pp. 959-976, 2017.

[26] M. N. Jebur, B. Pradhan, and M. S. Tehrany, "Manifestation of LiDAR-derived parameters in the spatial prediction of landslides using novel ensemble evidential belief functions and support vector machine models in GIS," IEEE J. Sel. Top. Appl. Earth Obs. Remote Sens., vol. 8, no. 2, pp. 674-690, 2015.

[27] B. A. S. Aji, T. H. Liong, and B. Muslim, "Detection precursor of sumatra earthquake based on ionospheric total electron content anomalies using N-Model Articial Neural Network," 2017 Int. Conf. Adv. Comput. Sci. Inf. Syst. ICACSIS 2017, vol. 2018-Janua, pp. 269-276, 2018.

[28] G. Tas, O. K. Ersoy, and M. E. Kamas, "SUPPORT VECTOR SELECTION AND ADAPTATION FOR CLASSIFICATION OF Istanbul Technical Univ, Informatics Institute , Istanbul, Turkey , e-mail: gulsen@be.itu.edu.tr Purdue University , School of Electrical and Computer Eng., Indiana ,USA, e-mail : ersoy@p," Computer (Long. Beach. Calif)., pp. 851-854, 2009.

[29] T. Perol, M. Gharbi, and M. Denolle, "Convolutional neural network for earthquake detection and location," Sci. Adv., vol. 4, no. 2, pp. 2-10, 2018.

[30] B. Gokaraju, A. C. Turlapaty, D. A. Doss, R. L. King, and N. H. Younan, "Change detection analysis of tornado disaster using conditional copulas and Data Fusion for cost-effective disaster management," 2015 IEEE Appl. Imag. Pattern Recognit. Work.
AIPR 2015, no. October, 2016.

[31] T. Sakaki, M. Okazaki, and Y. Matsuo, "Tweet analysis for realtime event detection and earthquake reporting system development," IEEE Trans. Knowl. Data Eng., vol. 25, no. 4, pp. 919-931, 2013.

[32] M. Wieland, W. Liu, and F. Yamazaki, "Learning change from Synthetic Aperture Radar images: Performance evaluation of a Support Vector Machine to detect earthquake and tsunami-induced changes," Remote Sens., vol. 8, no. 10, 2016.

[33] A. J. Cooner, Y. Shao, and J. B. Campbell, "Detection of urban damage using remote sensing and machine learning algorithms: Revisiting the 2010 Haiti earthquake," Remote Sens., vol. 8, no. $10,2016$.

[34] W. Li, N. Narvekar, N. Nakshatra, N. Raut, B. Sirkeci, and J. Gao, "Seismic data classification using machine learning," Proc. - IEEE 4th Int. Conf. Big Data Comput. Serv. Appl. BigDataService 2018, pp. 56-63, 2018.

[35] S. Park, J. Im, E. Jang, and J. Rhee, "Drought assessment and monitoring through blending of multi-sensor indices using machine learning approaches for different climate regions," Agric. For. Meteorol., vol. 216, pp. 157-169, 2016.

[36] V. Q. Nguyen, H. J. Yang, K. Kim, and A. R. Oh, "Real-Time Earthquake Detection Using Convolutional Neural Network and Social Data," Proc. - 2017 IEEE 3rd Int. Conf. Multimed. Big Data, BigMM 2017, pp. 154-157, 2017.

[37] M. Imran, S. Elbassuoni, C. Castillo, F. Diaz, and P. Meier, "Practical extraction of disaster-relevant information from social media," WWW 2013 Companion - Proc. 22nd Int. Conf. World Wide Web, pp. 1021-1024, 2013.

[38] S. N. K. B. Amit and Y. Aoki, "Disaster detection from aerial imagery with convolutional neural network," Proc. - Int. Electron. Symp. Knowl. Creat. Intell. Comput. IES-KCIC 2017, vol. 2017Janua, no. July 2018, pp. 239-245, 2017.

[39] M. Bria, A. Baban, and D. Dumitrascu, "A Region Thesaurus Approach for High-Level Concept Detection in the Natural Disaster Domain," An Interdiscip. J., vol. 16, no. 3, pp. 423-452, 2013.

[40] A. L. Pyayt, I. I. Mokhov, B. Lang, V. V. Krzhizhanovskaya, and R. J. Meijer, "Machine learning methods for environmental monitoring and flood protection," World Acad. Sci. Eng. Technol., vol. 78, no. 6, pp. 118-123, 2011.

[41] K. Kireyev, L. Palen, and K. Anderson, "Applications of topics models to analysis of disaster-related twitter data," NIPS Work. Appl. Top. Model. Text Beyond, p. , 2009.

[42] I. Yilmaz, "Comparison of landslide susceptibility mapping methodologies for Koyulhisar, Turkey: Conditional probability, logistic regression, artificial neural networks, and support vector machine," Environ. Earth Sci., vol. 61, no. 4, pp. 821-836, 2010.

[43] M. Scharkow, "Thematic content analysis using supervised machine learning: An empirical evaluation using German online news," Qual. Quant., vol. 47, no. 2, pp. 761-773, 2013.

[44] S. Ahmad, K. Ahmad, N. Ahmad, and N. Conci, "Convolutional neural networks for disaster images retrieval," CEUR Workshop Proc., vol. 1984, pp. 2016-2018, 2017.

[45] K. Ahmad, P. Konstantin, M. Riegler, N. Conci, and P. Holversen, "CNN and GAN based satellite and social media data fusion for disaster detection," CEUR Workshop Proc., vol. 1984, no. September, pp. 15-18, 2017.

[46] M. Maldonado, D. Alulema, D. Morocho, and M. Proano, "System for monitoring natural disasters using natural language processing in the social network Twitter," Proc. - Int. Carnahan Conf. Secur. Technol., no. October, 2017.

[47] D. Buscaldi and I. Hernandez-Farias, "Sentiment Analysis on Microblogs for Natural Disasters Management," Proc. 24th Int. Conf. World Wide Web - WWW'15 Companion, pp. 1185-1188, 2015.

[48] G. Shalunts, G. Backfried, and K. Prinz, "Sentiment Analysis of German Social Media Data for Natural Disasters," 11th Int. Conf. Inf. Syst. Cris. Response Manag., no. May, pp. 752-756, 2014.

[49] L. Maceda, J. Llovido, and A. Satuito, "Categorization of Earthquake-Related Tweets Using Machine Learning Approaches," no. 1, pp. 229-232, 2019.

[50] B. Truong, C. Caragea, A. Squicciarini, and A. H. Tapia, 
"Identifying valuable information from Twitter during natural disasters," Proc. ASIST Annu. Meet., vol. 51, no. 1, 2014.

[51] T. Valero, M. Gómez, and V. Pineda, "Using Machine Learning for Extracting Information from Natural Disaster News Reports," Comput. y Sist., vol. 13, no. 1, pp. 33-44, 2009.

[52] A. H. SALANS, J. A. SCHACK, and L. N. KATZ, "Natural Language Processing to the Rescue? Extracting 'Situational Awareness' Tweets During Mass Emergency," Circulation, vol. 2, no. 6, pp. 900-906, 1950.

[53] I. Varga et al., "Aid is out there: Looking for help from tweets during a large scale disaster," ACL 2013 - 51st Annu. Meet. Assoc. Comput. Linguist. Proc. Conf., vol. 1, pp. 1619-1629, 2013.

[54] C. Caragea, A. Silvescu, and A. H. Tapia, "Identifying informative messages in disaster events using Convolutional Neural Networks," Proc. Int. ISCRAM Conf., no. May, 2016.

[55] D. T. Nguyen, F. Ofli, M. Imran, and P. Mitra, "Damage Assessment from Social Media Imagery Data During Disasters," no. December, pp. 569-576, 2017.

[56] S. Cresci, M. Tesconi, A. Cimino, and F. Dell'Orletta, "A Linguistically-driven Approach to Cross-Event Damage Assessment of Natural Disasters from Social Media Messages," pp. 1195-1200, 2016.

[57] K. Zahra, F. O. Ostermann, and R. S. Purves, "Geographic variability of Twitter usage characteristics during disaster events," Geo-Spatial Inf. Sci., vol. 20, no. 3, pp. 231-240, 2017.

[58] Z. Huang, J. Zhou, L. Song, Y. Lu, and Y. Zhang, "Flood disaster loss comprehensive evaluation model based on optimization support vector machine," Expert Syst. Appl., vol. 37, no. 5, pp. 3810-3814, 2010.

[59] F. Ofli et al., "Combining Human Computing and Machine Learning to Make Sense of Big (Aerial) Data for Disaster Response," Big Data, vol. 4, no. 1, pp. 47-59, 2016.

[60] J. Peterson and B. Argo, "Tweedr: Mining Twitter to Inform Disaster Response," vol. 12, no. 4, pp. 354-358, 2014

[61] M. Imran, P. Mitra, and C. Castillo, "Twitter as a lifeline: Human- annotated Twitter corpora for NLP of crisis-related messages," Proc. 10th Int. Conf. Lang. Resour. Eval. Lr. 2016, pp. 1638-1643, 2016.

[62] J. R. Ragini, P. M. R. Anand, and V. Bhaskar, "Big data analytics for disaster response and recovery through sentiment analysis," Int. J. Inf. Manage., vol. 42, no. May, pp. 13-24, 2018.

[63] M. Avvenuti, F. Del Vigna, S. Cresci, A. Marchetti, and M. Tesconi, "Pulling Information from social media in the aftermath of unpredictable disasters," Proc. 2015 2nd Int. Conf. Inf. Commun. Technol. Disaster Manag. ICT-DM 2015, no. November, pp. 258-264, 2016.

[64] Y. Liu and L. Wu, "Geological Disaster Recognition on Optical Remote Sensing Images Using Deep Learning," Procedia Comput. Sci., vol. 91, no. Itqm, pp. 566-575, 2016.

[65] A. Cobo, D. Parra, and J. Navón, "Identifying Relevant Messages in a Twitter-based Citizen Channel for Natural Disaster Situations," 2015.

[66] H. Li et al., "Twitter Mining for Disaster Response: A Domain Adaptation Approach," 12th Int. Conf. Inf. Syst. Cris. Response Manag. (ISCRAM 2015), p. [1-7], 2015.

[67] S. Cresci, A. Cimino, F. Dell'Orletta, and M. Tesconi, "Crisis Mapping During Natural Disasters via Text Analysis of Social Media Messages," Lect. Notes Comput. Sci. (including Subser. Lect. Notes Artif. Intell. Lect. Notes Bioinformatics), vol. 9419, pp. 250-258, 2015.

[68] G. Beigi, R. Maciejewski, and H. Liu, "Sentiment Analysis and Ontology Engineering," vol. 639, no. February 2016, 2016.

[69] A. Fikri Aji, I. P. E. S. Putra, P. Mursanto, and S. Yazid, "Can smartphones be used to detect an earthquake? Using a machine learning approach to identify an earthquake event," 8th Annu. IEEE Int. Syst. Conf. SysCon 2014 - Proc., pp. 72-77, 2014.

[70] G. Rajguru, Y. S. Bhadauria, and S. Mukhopadhyay, "Estimation of Earthquake Source Parameters Using Machine Learning Techniques," 2018 9th Int. Conf. Comput. Commun. Netw. Technol. ICCCNT 2018, pp. 1-7, 2018. 


\section{CERTIFICATE}

This certificate is presented to

\section{Mr. Andi Wahju Rahardjo Eman-} uel

\section{as}

for outstanding contribution in the 4th 2019 International Conference on Information Technology, Information System, and Electrical Engineering held in Yogyakarta, Indonesia on 20 - 21 November 2019

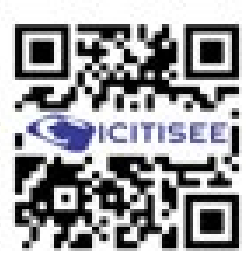

Conference No. \#48480

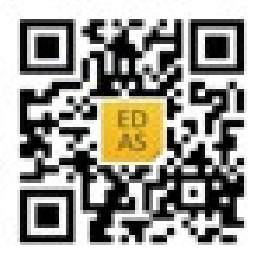

ID number 1129597

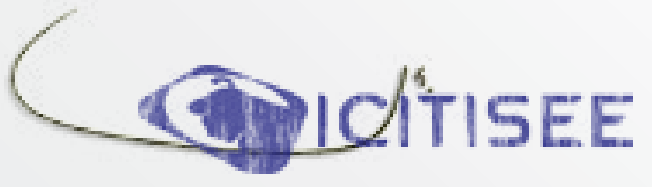

Dr. Kusrini, M.Kom.

General Chair

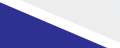

- IEEE INDONESIA SECTION
Department of mation Engineering Universitas Gadjah Mada 